\title{
Two-Dimensional Boundary Element Analysis of Edge Cracked Rectangular Plate Using Near-Tip Solution*
}

\author{
Toshio FURUKAWA** and Toshiyasu SUEYOSHI** \\ ${ }^{* *}$ Department of Mechanical Systems Engineering \\ University of the Ryukyus \\ 1 Senbaru, Nishihara, Okinawa 903-0213, Japan \\ E-mail: furukawa@teada.tec.u-ryukyu.ac.jp
}

\begin{abstract}
This paper is concerned with a method for the analysis of two-dimensional crack problems using the boundary element method. In this method, a domain to be analyzed is divided into two regions: the near-tip region, which is a circular in shape and centered at the crack tip, and the outer region. The displacements and stresses in the near-tip region are expressed by the truncated infinite series of the eigenfunction with unknown coefficients. The outer region is formulated by the boundary element method. The system of linear algebraic equations with respect to nodal displacements, nodal tractions and the coefficients of the series are constructed by discretized boundary integration in the outer region and the continuity conditions. The two parallel edge cracked rectangular plate without symmetry under a uniform tension is treated and the dimensionless stress intensity factors are calculated.
\end{abstract}

Key words: Computational Engineering, Fracture Mechanics, Elasticity, Boundary Element Method, Stress Intensity Factor, Near-Tip Solution, Slant Edge Crack, Domain Composition Method

\section{Introduction}

It is well known that Boundary Element Method (BEM) is more efficient method comparing with Finite Element Method (FEM) and Finite Difference Method (FDM) for elastic problems. However, some ideas are necessary in the crack problem to obtain an appropriate solution as well as the other numerical calculation methods because the stresses tend to infinity near the crack-tip, that is, stress singularity. There are a lot of methods to obtain the stress intensity factors (SIFs), such as, the extrapolation methods based on stress and displacement near the crack-tip ${ }^{(1),(2)}$ and the method introducing the singular element $^{(3)-(7)}$. The element division near the crack-tip is required in these methods and severe ideas are required to obtain the good accurate solution.

Authors proposed the method by which the stress intensity factors could be obtained in good accuracy without discretization near the crack-tip ${ }^{(8)}$. A virtual region including the crack-tip was introduced, and within the virtual region, near-tip infinite series solutions for displacements and stresses that satisfied the governing equations and the boundary conditions on the crack surface were applied and a usual boundary element method was used in other region. At the boundary of the real and virtual regions, the continuity conditions of displacements and stresses were introduced. The stress intensity factors were obtained by the simultaneous equations led from the discretized boundary integral equations. To test the accuracy of this method the center slant cracked rectangular plate 
under a uniform tension with point symmetry has been analyzed. The calculated dimensionless stress intensity factors were in good accuracy. Next, we extended this method to the cracked problem with no symmetry (edge cracked plate) by use of domain decomposition method, and showed the effectiveness of this proposed method ${ }^{(9),(10)}$. Now, we treat the two edge cracked rectangular plate and examine several cases.

\section{Analysis}

The near-tip solutions of displacements $u_{x}, u_{y}$ and stresses $\sigma_{x}, \sigma_{y}, \tau_{x y}$ for two-dimensional elastic body with crack are well known and expressed as follows ${ }^{(11)}$ :

$$
\begin{aligned}
& \left\{\begin{array}{l}
u_{x} \\
u_{y}
\end{array}\right\}=\sum_{n=1}^{\infty} \frac{A_{\mathrm{I} n}}{2 \mu} r^{n / 2}\left\{\begin{array}{c}
f_{\mathrm{I} n}^{(1)}(\theta) \\
f_{\mathrm{I} n}^{(2)}(\theta)
\end{array}\right\}-\sum_{n=1}^{\infty} \frac{A_{\mathrm{II} n}}{2 \mu} r^{n / 2}\left\{\begin{array}{l}
f_{\mathrm{II} n}^{(1)}(\theta) \\
f_{\mathrm{II} n}^{(2)}(\theta)
\end{array}\right\}+\left\{\begin{array}{l}
u_{x 0} \\
u_{y 0}
\end{array}\right\} \\
& \left\{\begin{array}{l}
\sigma_{x} \\
\sigma_{y} \\
\tau_{x y}
\end{array}\right\}=\sum_{n=1}^{\infty} A_{\mathrm{II} n} \frac{n}{2} r^{(n / 2)-1}\left\{\begin{array}{l}
g_{\mathrm{I} n}^{(1)}(\theta) \\
g_{\mathrm{I} n}^{(2)}(\theta) \\
g_{\mathrm{I} n}^{(3)}(\theta)
\end{array}\right\}-\sum_{n=1}^{\infty} A_{\mathrm{II} n} \frac{n}{2} r^{(n / 2)-1}\left\{\begin{array}{l}
g_{\mathrm{II} n}^{(1)}(\theta) \\
g_{\mathrm{II} n}^{(2)}(\theta) \\
g_{\mathrm{II} n}^{(3)}(\theta)
\end{array}\right\}
\end{aligned}
$$

where $f_{\mathrm{I} n}^{(i)}, f_{\mathrm{II} n}^{(i)}, g_{\mathrm{I} n}^{(i)}$ and $g_{\mathrm{II} n}^{(i)}$ are known functions of the angle $\theta$ as

$$
\begin{aligned}
& f_{\text {I } n}^{(1)}(\theta)=\kappa \cos \frac{n}{2} \theta-\frac{n}{2} \cos \left(\frac{n}{2}-2\right) \theta+\left\{\frac{n}{2}+(-1)^{n}\right\} \cos \frac{n}{2} \theta \\
& f_{\mathrm{I} n}^{(2)}(\theta)=\kappa \sin \frac{n}{2} \theta+\frac{n}{2} \sin \left(\frac{n}{2}-2\right) \theta-\left\{\frac{n}{2}+(-1)^{n}\right\} \sin \frac{n}{2} \theta \\
& f_{\mathrm{II} n}^{(1)}(\theta)=\kappa \sin \frac{n}{2} \theta-\frac{n}{2} \sin \left(\frac{n}{2}-2\right) \theta+\left\{\frac{n}{2}-(-1)^{n}\right\} \sin \frac{n}{2} \theta \\
& f_{\mathrm{II} n}^{(2)}(\theta)=-\kappa \cos \frac{n}{2} \theta-\frac{n}{2} \cos \left(\frac{n}{2}-2\right) \theta+\left\{\frac{n}{2}-(-1)^{n}\right\} \cos \frac{n}{2} \theta \\
& g_{\mathrm{I} n}^{(1)}(\theta)=\left\{2+(-1)^{n}+\frac{n}{2}\right\} \cos \left(\frac{n}{2}-1\right) \theta-\left(\frac{n}{2}-1\right) \cos \left(\frac{n}{2}-3\right) \theta \\
& g_{\mathrm{I} n}^{(2)}(\theta)=\left\{2-(-1)^{n}-\frac{n}{2}\right\} \cos \left(\frac{n}{2}-1\right) \theta+\left(\frac{n}{2}-1\right) \cos \left(\frac{n}{2}-3\right) \theta \\
& g_{\mathrm{I} n}^{(3)}(\theta)=-\left\{(-1)^{n}+\frac{n}{2}\right\} \sin \left(\frac{n}{2}-1\right) \theta+\left(\frac{n}{2}-1\right) \sin \left(\frac{n}{2}-3\right) \theta \\
& g_{\mathrm{II} n}^{(1)}(\theta)=\left\{2-(-1)^{n}+\frac{n}{2}\right\} \sin \left(\frac{n}{2}-1\right) \theta-\left(\frac{n}{2}-1\right) \sin \left(\frac{n}{2}-3\right) \theta \\
& g_{\mathrm{II} n}^{(2)}(\theta)=\left\{2+(-1)^{n}-\frac{n}{2}\right\} \sin \left(\frac{n}{2}-1\right) \theta+\left(\frac{n}{2}-1\right) \cos \left(\frac{n}{2}-3\right) \theta \\
& g_{\mathrm{II} n}^{(3)}(\theta)=-\left\{(-1)^{n}-\frac{n}{2}\right\} \cos \left(\frac{n}{2}-1\right) \theta-\left(\frac{n}{2}-1\right) \cos \left(\frac{n}{2}-3\right) \theta
\end{aligned}
$$

$A_{\mathrm{I} n}$ and $A_{\mathrm{II} n}$ are unknowns determined from crack configuration and loading condition, $\mu$ is rigidity, $u_{x 0}$ and $u_{y 0}$ are rigid body displacements of $x$ direction and $y$ direction, respectively. $\kappa$ is constant which is different by plane stress and plane strain.

The stress intensity factors are expressed as

$$
K_{\mathrm{I}}=\sqrt{2 \pi} A_{\mathrm{I} 1}, \quad K_{\mathrm{II}}=-\sqrt{2 \pi} A_{\mathrm{II} 1}
$$


The tractions $t_{x}$ and $t_{y}$ are expressed as follows.

$$
\left\{\begin{array}{l}
t_{x} \\
t_{y}
\end{array}\right\}=\sum_{n=1}^{\infty} A_{\mathrm{I} n} \frac{n}{2} r^{(n / 2)-1}\left\{\begin{array}{l}
h_{\mathrm{I} n}^{(1)}(\theta) \\
h_{\mathrm{I} n}^{(2)}(\theta)
\end{array}\right\}-\sum_{n=1}^{\infty} A_{\mathrm{II} n} \frac{n}{2} r^{(n / 2)-1}\left\{\begin{array}{l}
h_{\mathrm{II} n}^{(1)}(\theta) \\
h_{\mathrm{II} n}^{(2)}(\theta)
\end{array}\right\}
$$

where

$$
h_{\text {in }}^{(1)}(\theta)=g_{\text {in }}^{(1)}(\theta) \cos \theta+g_{\text {in }}^{(3)}(\theta) \sin \theta, \quad h_{\text {in }}^{(2)}(\theta)=g_{\text {in }}^{(2)}(\theta) \sin \theta+g_{\text {in }}^{(3)} \cos \theta
$$

We truncate the infinite series of Eqs. (1) and (4) by $N$. It is as follows when the results are denoted by the matrix form.

$$
\left\{\mathbf{U}^{\prime}\right\}=\left\{\begin{array}{l}
u_{x} \\
u_{y}
\end{array}\right\}=\left[\begin{array}{c}
F^{(1)}(r, \theta) \\
F^{(2)}(r, \theta)
\end{array}\right]\{\mathbf{A}\}, \quad\left\{\mathbf{T}^{\prime}\right\}=\left\{\begin{array}{l}
t_{x} \\
t_{y}
\end{array}\right\}=\left[\begin{array}{c}
H^{(1)}(r, \theta) \\
H^{(2)}(r, \theta)
\end{array}\right]\{\mathbf{A}\}
$$

where the elements of $\{\mathbf{A}\}$ are consisted from unknowns $A_{\mathrm{I} n}(n=1,2, \cdots, N)$, and $A_{\mathrm{II} n}(n=1,2, \cdots, N)$. In the formula of displacements (6a), rigid body displacements $u_{x 0}$ and $u_{y 0}$ are included in the elements of $\{\mathbf{A}\}$. Function $F^{(i)}(r, \theta)$ is constituted combining $r, f_{\mathrm{I} n}^{(i)}(\theta)$, and $f_{\mathrm{I} n}^{(i)}(\theta)$, and function $H^{(i)}(r, \theta)$ is also constituted combining $r, h_{\mathrm{I} n}^{(i)}(\theta)$, and $h_{\mathrm{II} n}^{(i)}(\theta)$.

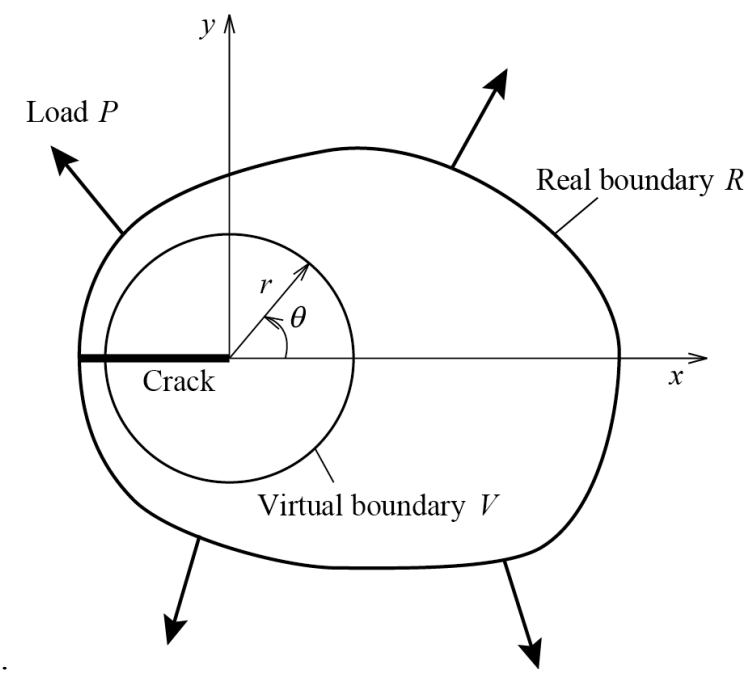

Fig. 1 Cracked body with virtual boundary

Now, we consider a cracked body as shown in Fig. 1. A virtual region, which is circular in shape and centered at the crack tip with radius $r$, is introduced. The discretized boundary integral equation for the body excluding the virtual circular region is

$$
\left[\begin{array}{ll}
\mathbf{H}_{R} & \mathbf{H}_{V}
\end{array}\right]\left\{\begin{array}{c}
\mathbf{U}_{R} \\
\mathbf{U}_{V}
\end{array}\right\}=\left[\begin{array}{ll}
\mathbf{G}_{R} & \mathbf{G}_{V}
\end{array}\right]\left\{\begin{array}{c}
\mathbf{T}_{R} \\
\mathbf{T}_{V}
\end{array}\right\}
$$

where $[\mathbf{H}]$ and $[\mathbf{G}]$ are coefficient for displacement $\{\mathbf{U}\}$ and traction $\{\mathbf{T}\}$, respectively. In this expression, the subscripts $R$ and $V$ denote the real boundary and virtual boundary, respectively.

The boundary conditions on virtual boundary are led from the continuity conditions for displacements and tractions and are expressed as

$$
\mathbf{U}_{i}^{\prime}-\mathbf{U}_{V i}=\mathbf{0}, \quad \mathbf{T}_{i}^{\prime}+\mathbf{T}_{V i}=\mathbf{0}
$$


where $\mathbf{U}_{i}^{\prime}$ and $\mathbf{T}_{i}^{\prime}$ are the discretized $i$-th term nodal displacement and traction of near-tip solutions, Eq. (6), respectively.

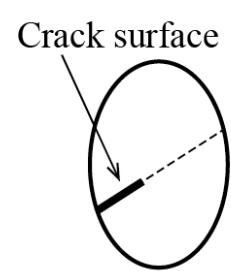

(a) Original Configuration

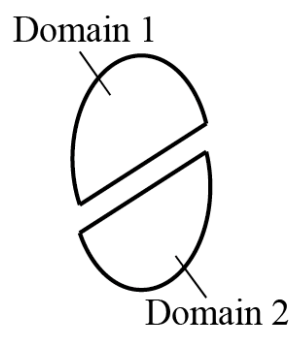

(b) Decomposition

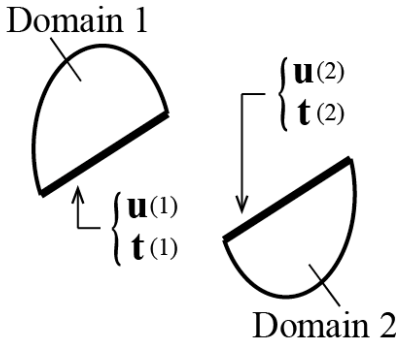

(c) Boundary conditions at a new interface

Fig. 2 Outline of domain decomposition

To treat the problem with no symmetry, we introduce the domain decomposition method as shown in Fig. 2. We decompose the original configuration to two domains along the crack surface. We combine the near-tip solutions and the usual boundary element method for domain 1 and domain 2, respectively. Then we use the combined condition of displacements and tractions

$$
\mathbf{u}(1)=\mathbf{u}(2), \quad \mathbf{t}(1)+\mathbf{t}(2)=\mathbf{0}
$$

where $\mathbf{u}(i)$ and $\mathbf{t}(i)$ are the boundary condition of displacements and tractions for domain $i$.

In Eq. (7), the unknowns of displacement and traction are summarized to the left hand side, and the known quantities are summarized to the right-hand side. As a result, the last equation turns into the usual simultaneous linear equations, and is as follows.

$$
[\mathbf{A}]\{\mathbf{x}\}=\{\mathbf{f}\}
$$

Here, vector $\{\mathbf{x}\}$ consists of unknowns of displacements and tractions, $[\mathbf{A}]$ is a coefficient matrix and $\{\mathbf{f}\}$ is a known vector.

\section{Numerical results and discussion}

We consider the edge cracked rectangular plate subjected to uniform tension $\sigma_{0}$ with width $W$ and height $H$, crack lengths are $a_{1}$ and $a_{2}$, the distance of these two cracks is $b$ as shown in Fig. 3. We decompose the plate to three domains and the boundary conditions of these domains are shown in the right hand side of this figure.

The dimensionless stress intensity factors are defined by

$$
F_{\mathrm{I} i}=\frac{K_{\mathrm{I} i}}{\sigma_{0} \sqrt{\pi a_{i}}}, \quad F_{\mathrm{II} i}=\frac{K_{\mathrm{II} i}}{\sigma_{0} \sqrt{\pi a_{i}}}
$$

We need to determine the parameters introduced in this method. 

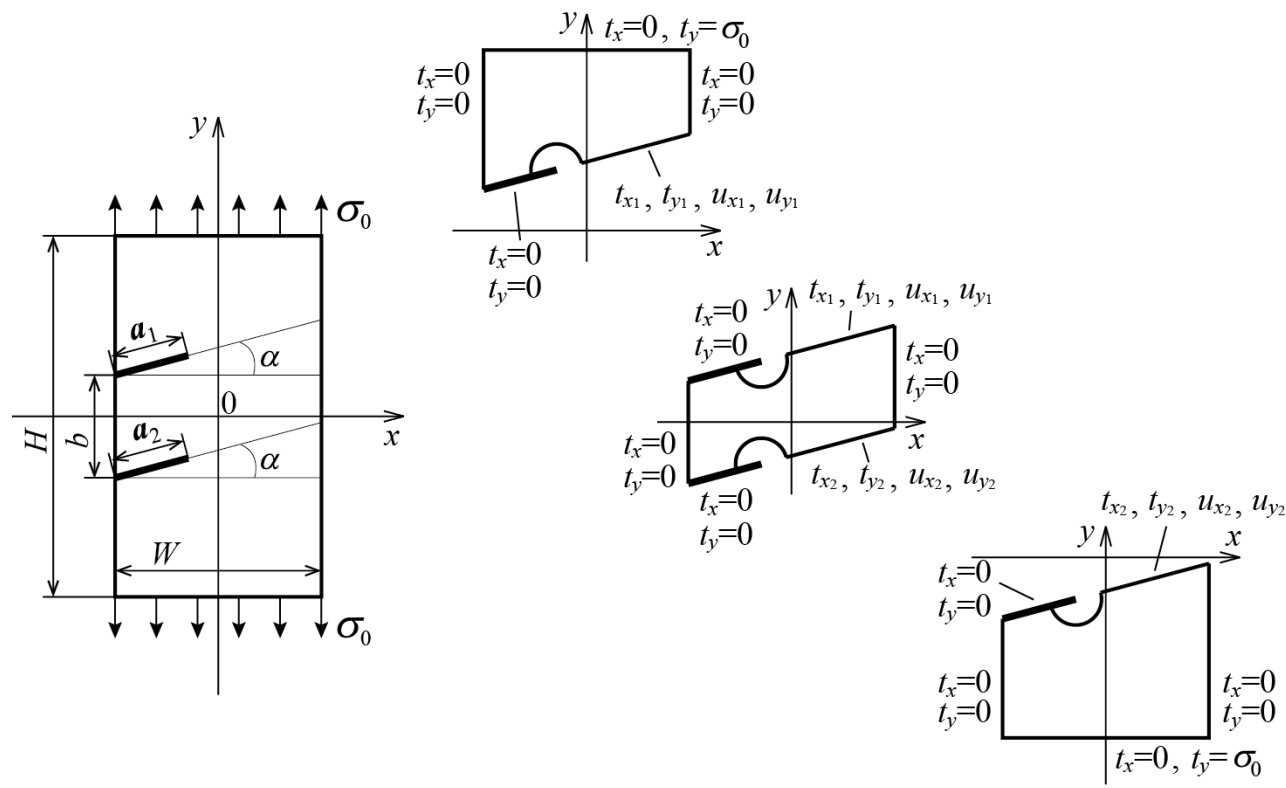

Fig. 3 Slant edge cracked rectangular plate and boundary conditions

\subsection{Truncated term number of infinite series of near-tip solution}

Firstly, we examine the truncated term number $N$ of infinite series. The common parameters are fixed that the crack angle $\alpha=\pi / 4$, total element number $M_{e}=1000$, slenderness ration $H / W=5$, crack length $A_{1}=a_{1} / W=0.25, A_{2}=a_{2} / W=0.25$, virtual radius $r_{1} / a_{1}=0.5, r_{2} / a_{2}=0.5$, and the crack distance $b / W=0.5$.

Figure 4 shows the relation of stress intensity factors and truncated term number $N$. The rhombus and square symbols mean the stress intensity factors for crack 1 and crack 2, respectively. The stress intensity factors are converged at $N=10$.

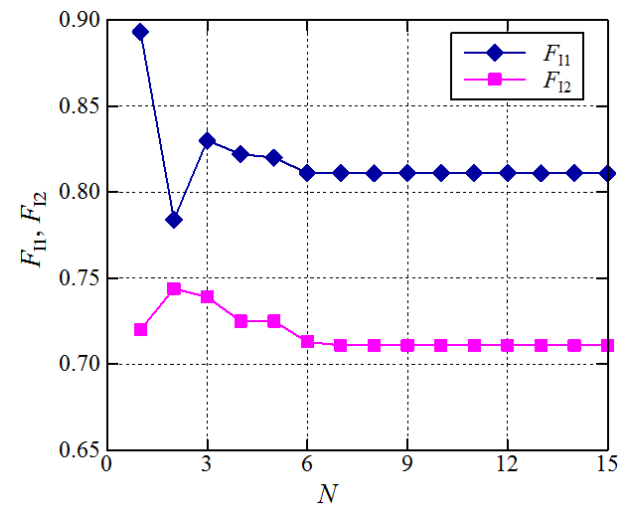

(a) Stress Intensity Factor $F_{\mathrm{I}}$

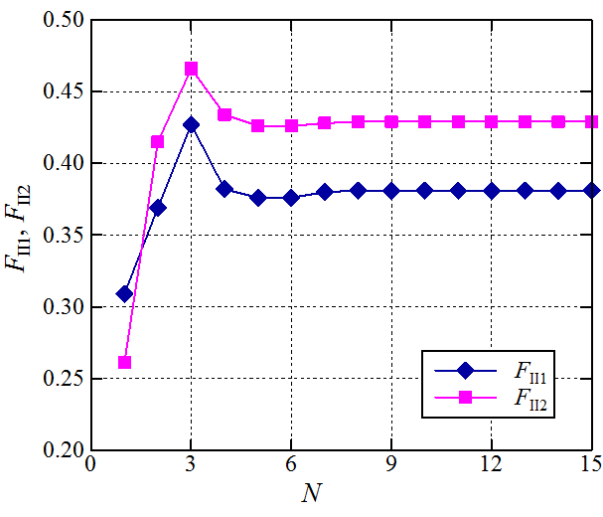

(b) Stress Intensity Factor $F_{\mathrm{II}}$

Fig. 4 Relations between stress intensity factors and term number of near-tip solution

\subsection{Total element number}

Next we examine the total element number $M e$. The remaining parameters are same as Section 3.1. Figure 5 shows the relation of stress intensity factors and total element number. The tendencies of four stress intensity factors are similar and the converged element number $M e$ is 1000 . 


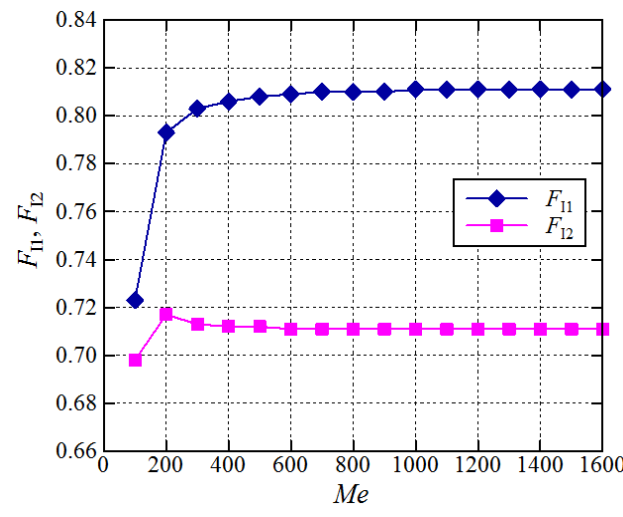

(a) Stress Intensity Factor $F_{\mathrm{I}}$

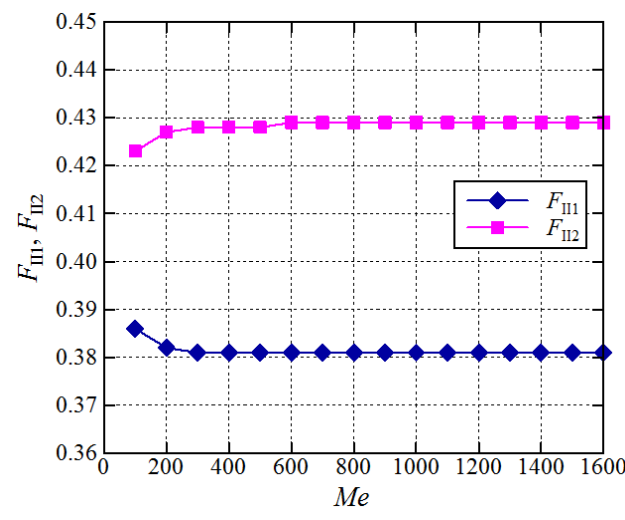

(b) Stress Intensity Factor $F_{\mathrm{II}}$

Fig. 5 Relations between stress intensity factors and total element number

\subsection{Slenderness ratio}

We examine the slenderness ratio $H / W$. Figure 6 shows the relation of stress intensity factors and slenderness ratio. The rhombus and square symbols mean the stress intensity factors for crack 1 and crack 2, respectively. The tendencies of four stress intensity factors are similar. When we put $H / W=5$, the plate is considered as the strip.

From these numerical calculations for the case that the crack angle $\alpha=\pi / 4$, the crack length $A_{1}=a_{1} / W=0.25, A_{2}=a_{2} / W=0.25$, and crack distance $b / W=0.5$, we adopt the parameters as that truncated term number $N=10$, total element number $M e=1000$, slenderness ratio $H / W=5$ and .virtual radius $r_{1} / a_{1}=0.5, r_{2} / a_{2}=0.5$.

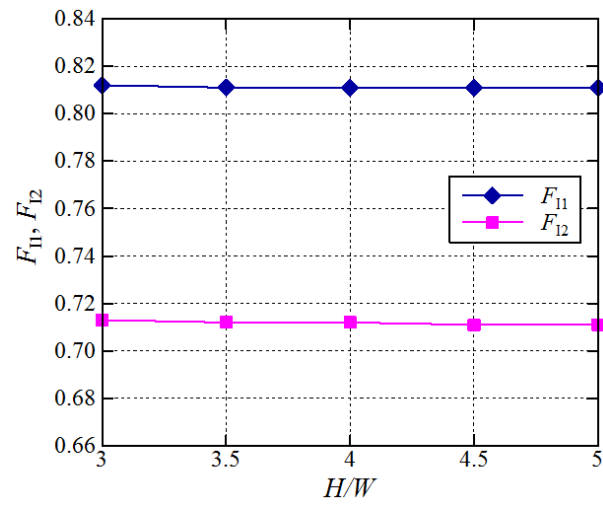

(a) Stress Intensity Factor $F_{I}$

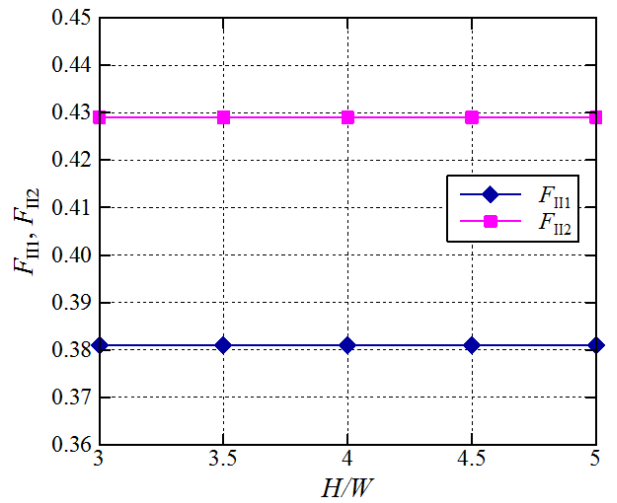

(b) Stress Intensity Factor $F_{\text {II }}$

Fig. 6 Relations between stress intensity factors and slenderness ratio

\subsection{Effect of distance of two cracks}

We examine the distance of two cracks $b / W$. For this section, the crack angle $\alpha=\pi / 4$, the crack lengths $A_{1}=a_{1} / W=0.25$, and $A_{2}=a_{2} / W=0.25$ are same as above-mentioned sections. Since two virtual regions will overlap if the distance of two cracks $b / W$ becomes 0.5 or less, the numerical value of the virtual radius $r_{1} / a_{1}=r_{2} / a_{2}$ is changed into $25 \%$ of the distance of two cracks. Figure 7 shows the relation of stress intensity factors and the distance of two cracks. The rhombus and square mean the stress intensity factors for crack 1 and crack 2 , respectively. The tendencies of four stress intensity factors are similar. The interference of two cracks occurs at $b / W<1.2$ and the stress intensity factor for $b / W>1.2$ is almost coincided to the stress intensity factor for single crack of the literature indicated in the stress intensity factor handbook ${ }^{(12)}$. 


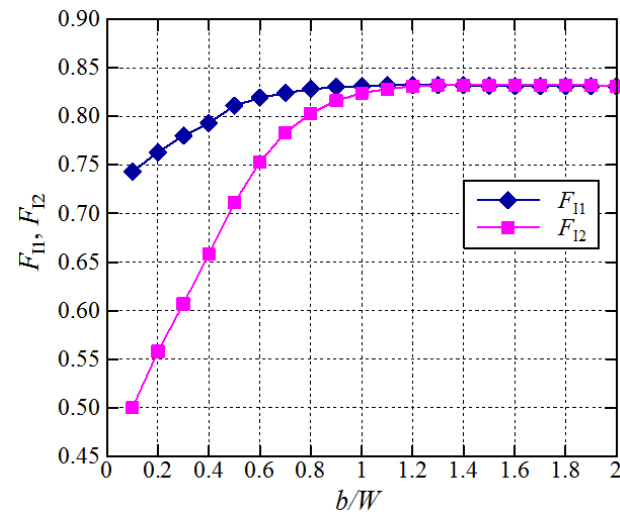

(a) Stress Intensity Factor $F_{\mathrm{I}}$

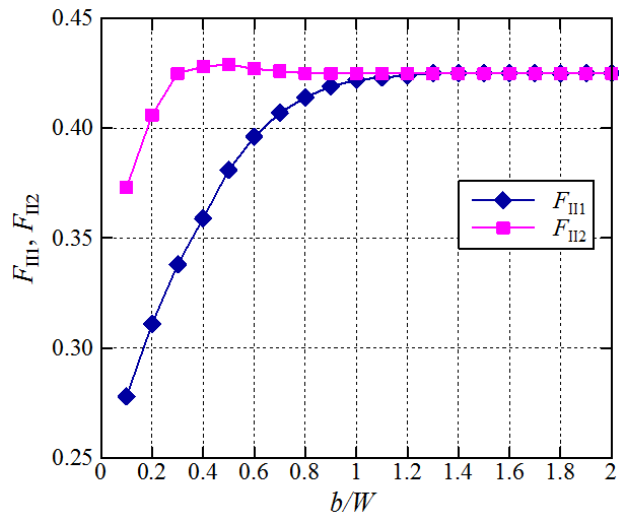

(b) Stress Intensity Factor $F_{\text {II }}$

Fig. 7 Relations between stress intensity factors and distance of two cracks

Next we examine that the limit value, which are the distance in which two cracks do not interfere, depends on the crack length, or not. Figure 8 shows the relations of stress intensity factors and the distance of two cracks for three cases. The rhombus, triangle and x-mark mean the stress intensity factors for $A_{1}=0.6, A_{1}=0.4$, and $A_{1}=0.2$ of crack 1 , respectively. The square, x-mark and circle mean the stress intensity factors for $A_{2}=0.6$, $A_{2}=0.4$, and $A_{2}=0.2$ of crack 2, respectively. From this figure, we know that the limit value depends on the crack length and the limit value becomes large when the crack length is longer.

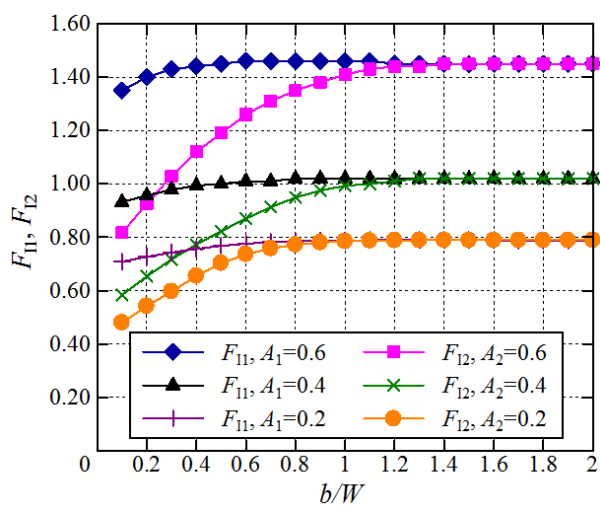

(a) Stress Intensity Factor $F_{\mathrm{I}}$

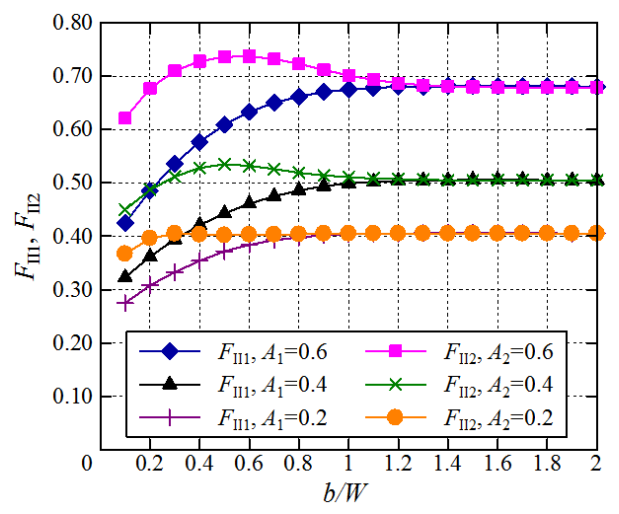

(b) Stress Intensity Factor $F_{\text {II }}$

Fig. 8 Relations between stress intensity factors and distance of two cracks for three cases

\subsection{Effect of crack length}

We examine the effect of crack length. For this section, the crack angle $\alpha=\pi / 4$ and distance of two cracks $b / W=0.5$ are same as above-mentioned sections. Figure 9 shows the relation of the stress intensity factors and crack length for $A_{1}=A_{2}$. The sign "c" of abscissa expresses crack length. The rhombus and square mean the stress intensity factors for crack 1 and crack 2, respectively. The difference of stress intensity factors of two cracks is small when the crack length is very short. The differences become monotonically large when the crack length is long.

Although illustration is omitted, the size of a virtual radius seldom influences a stress intensity factor. 


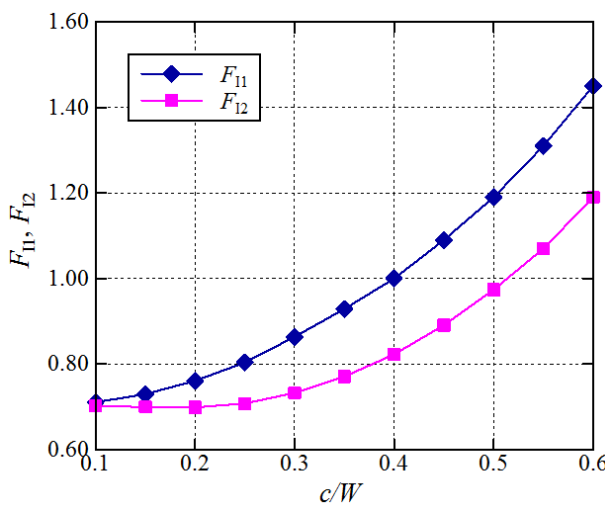

(a) Stress Intensity Factor $F_{\mathrm{I}}$

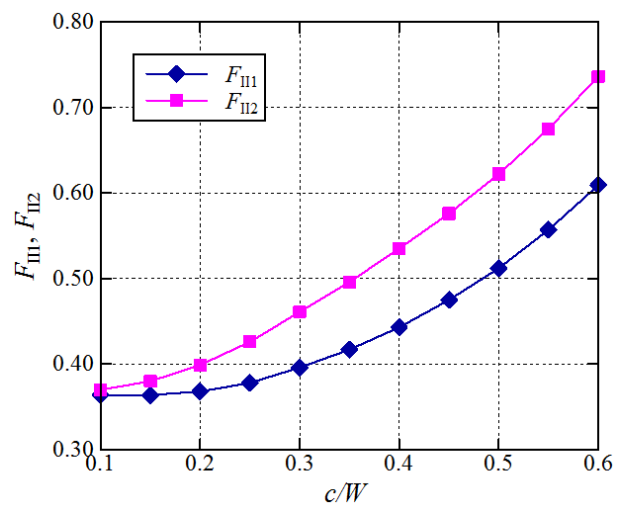

(b) Stress Intensity Factor $F_{\text {II }}$

Fig. 9 Relations between stress intensity factors and crack length

\section{Conclusion}

A highly accurate and simple solution method related to boundary element method has been presented. The numerical results discussed for two edge cracked rectangular plate with no symmetry are in good agreement with the available results in the literatures. Also the effects of various parameters such as plate width-to-height, crack length, truncated term number of near-tip infinite series solution, total number of boundary elements, and distance between two cracks are studied.

\section{Acknowledgements}

First author sincerely expresses his gratitude to the late professor Hiroshi Nakanishi of Kyoto Institute of Technology for the BEM solution methodology using near-tip solution.

\section{References}

(1) Tanaka, M., Hamada, M., and Iwata, Y., Computation of a Two-Dimensional Stress Intensity Factor by the Boundary Element Method, Ing.-Arch., Vol.52, No.1 (1982), pp.95-104.

(2) Furukawa, T., and Sueyoshi, T., Two Dimensional Elasticity Analyses of Materials with Defect by Boundary Element Method, Proc. of ACMFMS2012, Mechanics of Functional Materials and Structures, Kapuria, S., and Pradyumna, S. (Eds.), Narosa Publishing House, New Delhi (2012), pp.904-907.

(3) Liu, S. B., and Tan, C. L., Two-Dimensional Boundary Element Contact Mechanics Analysis of Angled Crack Problems, Eng. Fract. Mech., Vol.42, No.2 (1992), pp.273-288.

(4) Gao, Y. L. and Tan, C. L., Determination of Characterizing Parameters for Bimaterial Interface Cracks Using the Boundary Element Method, Eng. Fract. Mech., Vol.41, No.5 (1992), pp.779-784.

(5) Dong, Y., Wang, Z. and Wang, B., On the Computation Stress Intensity Factors for Interfacial Cracks Using Quarter-Point Boundary Elements, Eng. Fract. Mech., Vol.57 (1997), pp.335-342.

(6) Mukhopadhyay, N. K., Kakodkar, A., and Maiti, S. K., Further Considerations in Modified Crack Closure Integral Based Computation of Stress Intensity Factor in BEM, Eng. Fract. Mech., Vol.59, No.3 (1998), pp.269-279.

(7) Mukhopadhyay, N.K., Maiti, S.K., and Kakodkar, A., Effect of Modelling of Traction and Thermal Singularities on Accuracy of SIFs Computation through Modified Crack Closure Integral in BEM, Eng. Fract. Mech., Vol.64, No.2 (1999), pp.141-159.

(8) Nakanishi, H., Furukawa, T., and Honda, T., A Method of Analysis for Two-Dimensional 
Crack Problems by the Boundary Element Method, Transactions of the Japan Society of Mechanical Engineers, Vol.70A, No.692 (2004), pp.560-566.

(9) Furukawa, T., Two-dimensional Boundary Element Analysis of Edge Cracked Plate using Near-Tip Solution, Systems Modeling and Simulation, Theory and Applications, Asia Simulation Conference 2006, Koyamada, K., Tamura, S., and Ono, O. (Eds.), Springer, Tokyo (2006), pp.58-62.

(10)Furukawa, T., Boundary Element Analysis of Two-Dimensional Elastic Cracked Body Using Near-Tip Solution, Recent Advances in Computational Mechanics and Simulations, I. K. International, New Delhi, Vol. I (2006), pp.348-354.

(11) Okamura, H., Introduction to Linear Fracture Mechanics, Baifukan, Tokyo (1976), p.196.

(12) Murakami, Y., et al. (Eds.), Stress Intensity Factors Handbook, Pergamon, Oxford, Vol. 1 (1987), p.909. 